\title{
Role of Sorption Isotherms in the Analysis of Coupled Heat and Mass Fluxes in Porous Media
}

\author{
A. Heitor Reis and Rui Rosa \\ Geophysics Center of Évora and Department of Physics, University of Évora, \\ R. Romao Ramalho, 59, 7000-671 Évora, Portugal \\ E-mail: ahr@uevora.pt; rrosa@uevora.pt
}

\begin{abstract}
The aim of this work is to show the importance of the sorption isotherms in the study of the heat and mass fluxes in unsaturated porous media. General forms of the heat and mass fluxes are presented in terms of experimentally accessible quantities. The role of the isotherm slope in the coupling of heat and mass fluxes and its influence on the effective permeability are shown. Separate relations for vapor and liquid fluxes through the porous medium are presented as functions of the temperature and the isotherm slopes. Nonstationary isothermal mass flux is also analyzed, a relaxation time for this process is identified, and its relation to the isotherm slope is also discussed.
\end{abstract}

Received November 14, 2002; Accepted September 16, 2003

Copyright $\odot 2005$ Begell House, Inc. 


\section{NOMENCLATURE}

\begin{tabular}{|c|c|c|c|}
\hline$C_{p}$ & specific heat of the vapor, $\mathrm{J} \cdot \mathrm{kg}^{-1} \cdot \mathrm{K}^{-1}$ & $q$ & wave number, $\mathrm{m}^{-1}$ \\
\hline \multirow[t]{2}{*}{$D_{v}$} & coefficient of vapor diffusion within & $r$ & pore radius, $\mathrm{m}$ \\
\hline & the porous medium, $\mathrm{m}^{2} \cdot \mathrm{s}^{-1}$ & $S_{r}$ & saturation ratio \\
\hline$h$ & enthalpy per unit mass, $\mathrm{J} \cdot \mathrm{kg}^{-1}$ & $R_{\alpha}$ & specific gas constant, $\mathrm{J} \cdot \mathrm{kg}^{-1} \cdot \mathrm{K}^{-1}$ \\
\hline$\Delta h$ & enthalpy of condensation, ${\mathrm{J} \cdot \mathrm{kg}^{-1}}^{-1}$ & $\dot{s}$ & entropy generation per unit time, \\
\hline$K$ & permeability, $\mathrm{m}^{2}$ & & $\mathrm{~J} \cdot \mathrm{K}^{-1} \cdot \mathrm{s}^{-1}$ \\
\hline$K_{\text {eff }}$ & effective permeability, $\mathrm{m}^{2}$ & $t$ & time coordinate, $\mathrm{s}$ \\
\hline$k_{T}$ & thermal conductivity, $\mathrm{W} \cdot \mathrm{m}^{-1} \cdot \mathrm{K}^{-1}$ & $T$ & temperature, $\mathrm{K}$ \\
\hline $\mathbf{J}_{\mathrm{m}}$ & mass flux, $\mathrm{kg} \cdot \mathrm{m}^{-2} \cdot \mathrm{s}^{-1}$ & $x, y, z$ & spatial coordinates, $\mathrm{m}$ \\
\hline $\mathbf{J}_{\mathrm{mv}}$ & $\begin{array}{l}\text { mass flux in the vapor phase, } X, Y, Z \\
\mathrm{~kg} \cdot \mathrm{m}^{-2} \cdot \mathrm{s}^{-1}\end{array}$ & $X, Y, Z$ & dimensions, $\mathrm{m}$ \\
\hline \multirow[t]{2}{*}{$\mathbf{J}_{\mathrm{ml}}$} & mass flux in the liquid phase, & Greek symbols & \\
\hline & $\mathrm{kg} \cdot \mathrm{m}^{-2} \cdot \mathrm{s}^{-1}$ & $\gamma$ & surface tension, $\mathrm{Pa} \cdot \mathrm{m}$ \\
\hline $\mathbf{J}_{\mathbf{Q}}$ & heat flux, $\mathrm{W} \cdot \mathrm{m}^{-2}$ & $v$ & kinematic viscosity of the liquid, \\
\hline \multicolumn{3}{|l|}{$L_{m m}, L_{Q m}$} & $\mathrm{~m}^{2} \cdot \mathrm{s}^{-1}$ \\
\hline$L_{m Q}, L_{Q Q}$ & Onsager's coefficients & $\psi_{\alpha}$ & mass concentration of fluid $\alpha$ \\
\hline \multirow[t]{3}{*}{$M_{T S}$} & ratio between temperature driven & & within the porous medium, $\mathrm{kg} \cdot \mathrm{m}^{-3}$ \\
\hline & mass flux and humidity & $\chi\left(\theta_{P}, t, x, 0,0\right)$ & function that modulates \\
\hline & driven mass flux & & the evolution of the mass flux \\
\hline \multirow[t]{2}{*}{ QST } & ratio between humidity driven heat & $\rho$ & vapor density, $\mathrm{kg} \cdot \mathrm{m}^{-3}$ \\
\hline & flux and temperature driven heat flux & $\rho_{1}$ & liquid density, $\mathrm{kg} \cdot \mathrm{m}^{-3}$ \\
\hline$\dot{Q}$ & heat generation per unit time, $\mathrm{J} \cdot \mathrm{m}^{-3} \cdot \mathrm{s}^{-1}$ & $\tau$ & relaxation time, $\mathrm{s}$ \\
\hline$P$ & pressure, $\mathrm{Pa}$ & $\vartheta$ & liquid/surface contact angle, rad \\
\hline$P_{s}$ & saturation pressure, $\mathrm{Pa}$ & $\theta_{P}$ & isotherm slope, $\mathrm{kg} \cdot \mathrm{m}^{-3} \cdot \mathrm{Pa}^{-1}$ \\
\hline
\end{tabular}

\section{INTRODUCTION}

Porous media are open systems that can exchange heat and mass with their surroundings. In fact, a porous medium can take up relatively large amounts of gas condensed on the surface of the pores (adsorption) and may also have its void space partially or totally filled with a liquid phase (sorption). Gas adsorption on the pore inner surface of mesoporous materials takes place at low subsaturation $\left(P / P_{s}\right)<0.3$. As the gas pressure increases, the pores become progressively filled with the liquid phase. Adsorption involves phase change enthalpies, which are usually higher than condensation enthalpies, and, in general, the mass flux inside unsaturated porous media occurs in both the liquid and vapor phases. In this way, heat and mass fluxes in porous media are strongly coupled. The treatment of these processes in the usual framework of mechanics and thermodynamics is not an easy task, in part, because of difficulties in adequately modeling the system under study, but also because the quantities involved cannot be easily obtained from experiments. In addition, the porous media itself often develop internal stresses, strains, and even chemical reactions.

There are few consistent thermodynamic descriptions of these processes. Most of the published work on unsaturated porous media deals with the development of the 


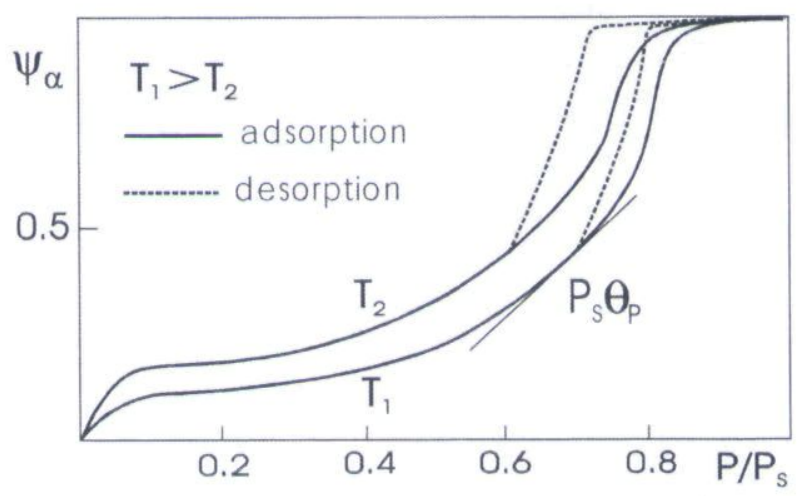

Figure 1. Typical sorption isotherms for a fluid within a mesoporous medium showing adsorption/desorption hysteresis.

theory of drying (Luikov, 1975; Whitaker, 1977; Ben Nasrallah and Perre, 1988). More recent theories of phase transitions in porous media developed by De Boer (1995) and De Boer and Kowalski (1995) provide some insight into the problem but are not of wide application. An extensive review of multiphase heat and mass flow in porous media may be found in Wang and Cheng (1997). Some years ago, Petit et al (1999) presented a model of two-phase flow based on local nonequilibrium conditions and Gray (2000) discussed the macroscopic equilibrium conditions for two-phase flow.

The problem of heat and mass fluxes in rigid porous media is analyzed in this work from a different perspective. The analysis is theoretically founded on the Onsager theory of the irreversible processes in relation to the phenomenological laws that involve experimentally measurable quantities. On the other hand, we take into account the work done in the field of the characterization of porous solids using physical adsorption. Sorption isotherms of gases and vapors are available for many kinds of porous materials and provide a lot of information, not only about the geometric properties of the inner surface (e.g., specific surface area, porosity, pore size distributions), but also about fluid/surface interaction.

The pores are void spaces in a solid matrix, and with the exception of the zeolitelike materials, the pore shape and dimensions vary within a wide range and the statistics of their widths is described by pore size distributions. Materials are usually classified according to their characteristic range of pore width. Microporous materials have pore widths around $2 \mathrm{~nm}$, mesoporous materials between $2 \mathrm{~nm}$ and $50 \mathrm{~nm}$, and macroporous materials have pore widths higher than $500 \mathrm{~nm}$.
In microporous materials the interaction potential (between the fluid and the pore surface) is highly enhanced owing to the proximity of the walls and gas adsorption. Pore filling usually takes place at very low subsaturation in this case (Gregg and Sing, 1982).

The mass concentration of a fluid $\alpha$ within a porous medium in equilibrium with its vapor at partial pressure $P$ and temperature $T_{0}$ is a function of pressure and temperature. The isotherms $\psi_{\alpha}\left(P, T_{0}\right)$, corresponding to a definite temperature $\mathrm{T} 0$, may be determined through adsorption experiments. In mesoporous materials, adsorption takes place at low saturation ratio $\left(P / P_{s}\right)<0.3$ but pore filling extends over a wide pressure range $0.3<\left(P / P_{s}\right)<1.0$ and complete filling occurs closely before the saturation pressure (see Fig. 1). The plateau at the end of the isotherm (close to $\left.\left(P / P_{s}\right)=1.0\right)$ corresponds to complete pore filling. All isotherms of the same fluid coincide at this plateau, the same being verified with different fluids when the amount adsorbed is expressed in terms of the equivalent volume of the liquid phase, following the Gurvitsch rule (Gregg and Sing, 1982).

In many mesoporous materials desorption isotherms do not coincide entirely with the respective sorption isotherms, showing a hysteresis loop in the part corresponding to pore filling. The typical form of these adsorption/desorption isotherms is shown in Fig. 1 (see also Gregg and Sing, 1982). In microporous media it is also possible to map sorption isotherms, which do not show adsorption/desorption hysteresis. Because of the very low pore width of these materials, Knudsen diffusion and surface diffusion dominate over Fick diffusion.

In macroporous materials it is practically impossible to map out an isotherm since condensation only occurs very close to the saturation pressure. 
Therefore, as the theory presented bellow is mainly based on the isotherm properties and on Darcy and Fick's law, its applicability is restricted to unsaturated mesoporous media.

\section{GENERAL FORMS OF COUPLED HEAT AND MASS FLUXES IN POROUS MEDIA}

\subsection{Sorption Isotherms for a Mesoporous Medium}

Capillary condensation of the vapor phase within mesoporous materials begins at low subsaturation, following Kelvin's law (Gregg and Sing, 1982); that is, pores of radius $r$ become filled with the liquid phase of fluid $\alpha$ at the corresponding saturation ratio $S_{r}=P / P_{s}$

$$
\ln S_{r}=-\frac{2 \gamma \cos \vartheta}{r \rho_{l} R_{\alpha} T}
$$

Then, in general, the liquid and vapor phases coexist within porous materials in wide temperature and pressure ranges.

The wetting affinity to the pore surface is very different for each substance in the gaseous mixture within the porous medium. For example, in the ambient air, water is usually the fluid with higher wetting affinity and predominates within the porous media.

In the following, it is assumed that definite values of $P$ and $T$ may be ascribed to each point within the porous medium and when dealing with spatial variation of these variables, it is also assumed that local thermodynamic equilibrium exists and is described by the "macroscopic" sorption isotherm curves $\psi_{\alpha}\left(P, T_{0}\right)$.

Moreover, in this analysis only sorption isotherms corresponding to fluids whose "wetting affinity" is much greater than all other fluids in the mixture are considered. In such cases, the sorption isotherms are practically insensitive to the total ambient pressure as it happens with the water isotherms of porous materials in moist air at the atmospheric pressure.

For each sorption isotherm $\psi_{\alpha}\left(P, T_{0}\right)$, we have

$$
\nabla \psi_{\alpha}=\theta_{p} \nabla P
$$

where

$$
\theta_{P}=\left(\partial \psi_{\alpha} / \partial P\right)_{T_{0}}
$$

The variable $\theta_{P}$ provides important information about the thermodynamic state of the fluid within the porous media and will be used in the analysis of the heat and mass fluxes.
If temperature and pressure gradients do exist inside the porous medium, then the system will react producing heat and mass fluxes. The theory of the irreversible processes (Onsager) allows for the description of these fluxes in terms of a linear combination of the existing gradients that act as driving forces for the fluxes (Kondepudi and Prigogine, 1998).

\subsection{Coupling of Mass and Heat Fluxes as Described by Onsager Theory and Phenomenological Relations}

The heat and mass fluxes and the respective driving forces may be identified through the entropy production rate in a unit control volume (Kondepudi and Prigogine, 1998)

$$
\dot{s}=\mathbf{J}_{\mathbf{Q}} \cdot \nabla(1 / T)-\mathbf{J}_{m} \cdot \nabla(\mu / T)
$$

where $T$ is the temperature and $\mu$ is the chemical potential. In this equation, we identify $\nabla(1 / T)$ and $\nabla(\mu / T)$ as the driving forces for the heat flux $\mathbf{J}_{\mathrm{Q}}$ and the mass flux $\mathbf{J}_{\mathrm{m}}$, respectively.

Then, in the linear regime, these fluxes read

$$
\mathbf{J}_{\mathbf{m}}=-L_{m m} \nabla(\mu / T)+L_{m Q} \nabla(1 / T)
$$

and

$$
\mathbf{J}_{Q}=-L_{Q m} \nabla(\mu / T)+L_{Q Q} \nabla(1 / T)
$$

The mass flux may occur in both the vapor and liquid phases (see Fig. 2). If the partial pressure of the vapor in equilibrium with liquid at meniscus $\alpha$ is higher than vapor pressure at $\beta$, then vapor diffuses in the gas mixture from $\alpha$ to $\beta$.. The mass flux in the liquid phase is considered to be a Darcy flow. In Eq. (5) we do not separate liquid and vapor flow since the assumption of local thermodynamic equilibrium ensures that at the same point the temperature $T$ and the chemical potential $\mu$ is the same for both the liquid and vapor.

The Onsager's Reciprocity Theorem ensures that

$$
L_{m Q}=L_{Q m}
$$

Therefore, only three unknown coefficients remain to be determined from phenomenological relations that must allow these coefficients in terms of experimentally known parameters. Equations (5) and (6) form the adequate framework for the description of linearly coupled heat and mass fluxes within porous media. 


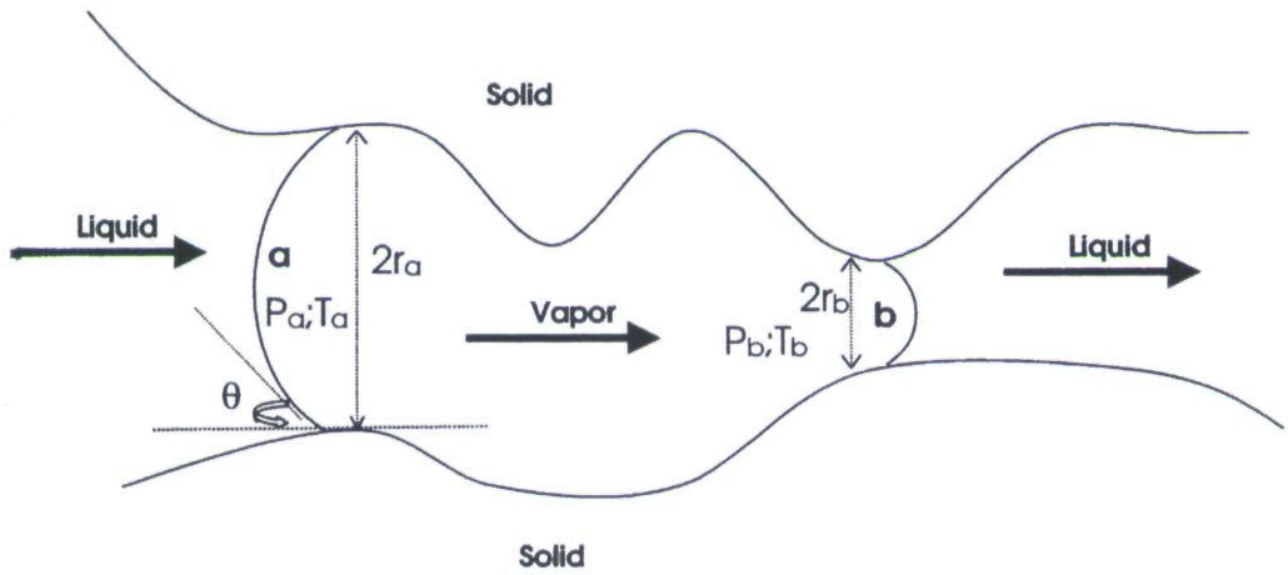

Figure 2. Schematic representation of the mass fluxes within a pore.

The unknown coefficients may be determined by considering the limiting situation of an isothermal porous media. As under the assumption of local equilibrium, the variation of $\mu, P$, and $T$ is the same for both the liquid and vapor phases, then by applying the Gibbs-Duhem equation to the vapor phase, $(1 / \rho) d P-d \mu=0$, Eq. (5) can be converted into

$$
\mathbf{J}_{\mathrm{m}}=-L_{m m}(\rho T)^{-1} \nabla P
$$

where $\rho$ stands for the density of the vapor phase. The coefficient $L_{m m}$ may be expressed in terms of known parameters with the help of the phenomenological relations describing the vapor and liquid components of the isothermal mass flux. The pertinent phenomenological relations are Darcy's law for the liquid:

$$
\mathbf{J}_{\mathrm{ml}}=-(K / \mathrm{v}) \nabla P
$$

and Fick's law, for the vapor:

$$
\mathbf{J}_{\mathrm{mv}}=-D_{v} \nabla \rho
$$

In the Eq. (9), K represents the permeability of the medium and $v$ the viscosity of the liquid; in the Eq. (10), $D_{v}$ stands for coefficient of vapor diffusion within the porous medium.

In the representative elementary volume of the unsaturated porous medium, vapor and mass flux coexist. The total mass flux is the sum of the fluxes of the liquid and vapor phases (see Appendix)

$$
\mathbf{J}_{\mathrm{m}}=-\left(K_{\mathrm{eff}} / v\right) \nabla P
$$

where $K_{\text {eff }}$ accommodates both the effect of convection (through $K$ ) and diffusion (through $D_{v}$ ).

Therefore, from Eqs. (8) and (11):

$$
L_{m m}=\left(K_{\text {eff }} \rho T\right) / v
$$

On the other hand, under isothermal conditions from the Eqs. (5) and (6) we get:

$$
\mathbf{J}_{\mathbf{Q}}=\frac{L_{Q m}}{L_{m m}} \mathbf{J}_{\mathrm{m}}
$$

For a stationary isothermal heat flux, applying the divergence operator to both members of this equation and combining with the energy conservation equation, $\rho C_{P}$ $(\partial T / \partial t)+\nabla \cdot J_{Q}=\dot{Q}$, one obtains:

$$
\dot{Q}=\frac{L_{Q m}}{L_{m m}} \nabla \cdot \mathbf{J}_{\mathbf{m}}+\nabla\left(\frac{L_{Q m}}{L_{m m}}\right)_{T} \cdot \mathbf{J}_{\mathbf{m}}
$$

Equation (14) describes an isothermal heat flux carried by a mass flux. Within porous media this may occur through the enthalpy carried by the vapor phase; that is,

$$
\left(\mathbf{J}_{Q}\right)_{\nabla T=0}=(\Delta h) \mathbf{J}_{\mathrm{mv}},
$$

where $\Delta h$ stands for the latent heat. Equation (14) shows that the divergence of the mass flux corresponds to condensation (or evaporation) of the vapor, therefore, releasing (or absorbing) latent heat. This process is described by

$$
\frac{\partial \rho}{\partial t}+\nabla \cdot \mathbf{J}_{\mathrm{mkv}}=\dot{Q} / \Delta h
$$


As to the coefficient $L_{Q m}$ it may be related to experimentally measurable quantities through the mass conservation equation

$$
\frac{\partial \psi_{\alpha}}{\partial t}+\nabla \cdot \mathbf{J}_{\mathrm{m}}=0
$$

which, combined with the Eqs. (3) and (16) and taking into account the ideal gas law, $P=\rho R_{\alpha} T$, gives

$$
\nabla .\left(\mathbf{J}_{\mathbf{m}}-\theta_{P} R_{\alpha} T \mathbf{J}_{\mathbf{m v}}\right)=-\dot{Q} \theta_{P} R_{\alpha} T / \Delta h
$$

Now, let $\dot{Q}=0$. Then, from Eqs. (15) and (18) results

$$
\mathbf{J}_{\mathbf{m}}=\left(\theta_{P} R_{\alpha} T\right) \mathbf{J}_{\mathbf{m v}}=\left(\theta_{P} R_{\alpha} T / \Delta h\right) \mathbf{J}_{\mathbf{Q}}
$$

and identifying similar terms in Eqs. (13) and (19) we arrive at

$$
\frac{L_{Q m}}{L_{m m}}=\frac{\Delta h}{\theta_{P} R_{\alpha} T}
$$

Finally, from the Eqs. (12) and (20) we obtain

$$
L_{Q m}=\frac{K_{\mathrm{eff}} \rho(\Delta h)}{v \theta_{P} R_{\alpha}}
$$

The coefficient $L_{Q Q}$ may in turn be obtained through Eqs. (5) and (6) by setting $\mathbf{J}_{\mathrm{m}}=0$, i.e.,

$$
\mathbf{J}_{\mathbf{Q}}=-\frac{1}{T^{2}}\left(L_{Q Q}-\frac{\left(L_{Q m}\right)^{2}}{L_{m m}}\right)(\nabla T)_{J_{m}=0}
$$

and making the identification with Fourier's law we have

$$
\frac{1}{T^{2}}\left(L_{Q Q}-\frac{\left(L_{Q m}\right)^{2}}{L_{m m}}\right)=k_{T}
$$

where $k_{T}$ stands for the thermal conductivity of the porous medium plus fluid. Therefore, we have from the Eqs. (12), (21), and (23)

$$
L_{Q Q}=k_{T} T^{2}+\frac{K_{\text {eff }}(\Delta h)^{2} \rho}{v \theta_{P}^{2} R_{\alpha}^{2} T}
$$

Through Eqs. (12), (21), and (24) we have related the three unknown coefficients in Eqs. (5) and (6) to the effective permeability $K_{\text {eff }}$, the thermal conductivity of the porous medium plus fluid $k_{T}$, and the isotherm slope $\theta_{P}$, which can be experimentally determined. Equations (5) and (6), with the forms obtained for their four coefficients, read

$$
\begin{aligned}
& \mathbf{J}_{\mathbf{m}}=-\frac{K_{\text {eff }} \rho T}{v} \nabla(\mu / T)+\frac{K_{\text {eff }} \rho(\Delta h)}{v \theta_{P} R_{\alpha}} \nabla(1 / T) \\
& \mathbf{J}_{\mathbf{Q}}=-\frac{K_{\text {eff }} \rho(\Delta h)}{v \theta_{P} R_{\alpha}} \nabla(\mu / T) \\
& +\left(k_{T} T^{2}+\frac{K_{\text {eff }}(\Delta h)^{2} \rho}{v \theta_{P}^{2} R_{\alpha}^{2} T}\right) \nabla(1 / T)
\end{aligned}
$$

or with the help of the Gibbs-Duhem equation one may have these equations expressed in terms of $P$ and $T$

$$
\begin{aligned}
& \mathbf{J}_{\mathbf{m}}=-\frac{K_{\text {eff }}}{v} \nabla P \\
& +\frac{K_{\text {eff }} \rho}{v T}\left(h-\frac{\Delta h}{\theta_{P} R_{\alpha} T}\right) \nabla T \\
& \mathbf{J}_{\mathbf{Q}}=-\frac{K_{\text {eff }}(\Delta h)}{v \theta_{P} R_{\alpha} T} \nabla P \\
& -\left(k_{T}-\frac{K_{\text {eff }} \rho(\Delta h) h}{v \theta_{P} R_{\alpha} T^{2}}+\frac{K_{\text {eff }} \rho(\Delta h)^{2}}{v \theta_{P}^{2} R_{\alpha}^{2} T^{3}}\right) \nabla T
\end{aligned}
$$

where $h=u+P / \rho$ stands for the enthalpy per unit mass of the vapor, whereas the effective permeability is given by (see Appendix)

$$
K_{\text {eff }} / K=1-\left(\theta_{P} R_{\alpha} T\right)^{-1}+K^{-1} D_{v} v \theta_{P}^{-1} R_{\alpha}^{-2} T^{-2}
$$

The heat and mass fluxes may also be expressed in terms of the saturation ratio $S_{r}=P / P_{s}$. The saturation pressure $P_{S}$ being given by the Clausius-Clapeyron equation

$$
d \ln P_{s}=\frac{L}{R_{\alpha} T^{2}} d T
$$

Equations (27) and (28) read

$$
\begin{aligned}
& \mathbf{J}_{\mathbf{m}}=-\frac{K_{\text {eff }} \rho R_{\alpha} T}{v S_{r}} \nabla S_{r} \\
& -\frac{K_{\text {eff }} \rho(\Delta h)}{v T}\left(1+\frac{1}{\theta_{P} R_{\alpha} T}-\frac{h}{\Delta h}\right) \nabla T \\
& \mathbf{J}_{\mathbf{Q}}=-\frac{K_{\text {eff }} \rho(\Delta h)}{v \theta_{P} S_{r}} \nabla S_{r} \\
& -\left[k_{T}+\frac{K_{\text {eff }} \rho(\Delta h)^{2}}{v T} \frac{1}{\theta_{P} R_{\alpha} T}\left(1+\frac{1}{\theta_{P} R_{\alpha} T}-\frac{h}{\Delta h}\right)\right] \nabla T
\end{aligned}
$$

These equations allow for the assessment of the relative importance of the cross flows in heat and mass transfer in porous media. 


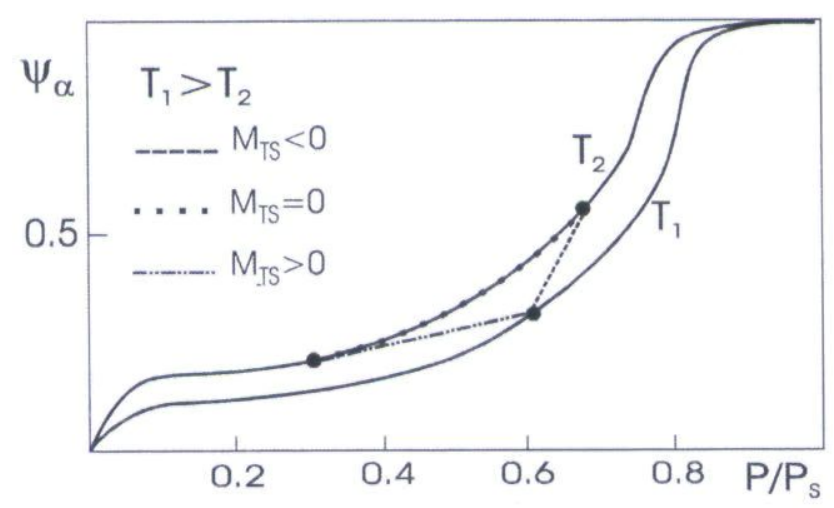

Figure 3. Ratios $M_{T S}$ for various mass fluxes within a mesoporous medium. For $M_{T S}<0$ the contributions of the temperature and of the saturation gradients have opposite signs and partially or totally cancel each other. For $M_{T S}=0$ the mass flux is totally driven by the saturation ratio gradient. For $M_{T S}>0$ both contributions have the same sign and add each other.

In order to further clarify the influence of the temperature and pressure gradients on the mass flux let $M_{T S}$ stand for the ratio between the part of the mass flux driven by the temperature gradient and the part driven by a gradient of the saturation ratio. Taking the enthalpy per unit mass of the vapor as

$$
h=C_{P} T
$$

we have from Eq. (31)

$$
M_{T S}=\left(1+\frac{1}{\theta_{p} R_{\alpha} T}-\frac{C_{P} T}{\Delta h}\right) \frac{\Delta h}{R_{\alpha} T} \frac{S_{r} \nabla T}{T \nabla S_{r}}
$$

In a similar way we determine the ratio $Q_{S T}$ between the part of the heat flux driven by the saturation ratio gradient and the part driven by the temperature gradient

$$
\begin{aligned}
& Q_{S T}=\left(1+\frac{1}{\theta_{P} R_{\alpha} T}-\frac{C_{P} T}{\Delta h}+\theta_{P} R_{\alpha} T \frac{k_{T} v T}{K_{\text {eff }} \rho(\Delta h)^{2}}\right)^{-1} \\
& \times \frac{R_{\alpha} T}{\Delta h} \frac{T \nabla S_{r}}{S_{r} \nabla T}
\end{aligned}
$$

For mesoporous media, $K$ is usually higher than $10^{-11} \mathrm{~m}^{2}$, $k_{T} \sim 1 \mathrm{~W} \cdot \mathrm{m}^{-1} \cdot \mathrm{K}^{-1}$ and with the fluid properties corresponding to $10^{\circ} \mathrm{C}$, the nondimensional factor $\left[k_{T} v T /\right.$ $\left.K_{\text {eff }} \rho(\Delta h)^{2}\right]$ is less than $10^{-5}$. The other nondimensional factor $\left(\theta_{P} R_{\alpha} T\right)$ appearing in Eq. (35) is normally smaller than $10^{2}$ (see Gregg and Sing, 1982). Therefore, most of the time the product of these two factors may be neglected in Eq. (35), which thus simply reads

$$
Q_{S T} \cong\left(1+\frac{1}{\theta_{P} R_{\alpha} T}-\frac{C_{P} T}{\Delta h}\right)^{-1} \frac{R_{\alpha} T}{\Delta h} \frac{T \nabla S_{r}}{S_{r} \nabla T}
$$

In this case, Eqs. (34) and (36) show that $M_{T S}$ is the inverse of $Q_{S T}$, i.e., $M_{T S} Q_{S T}=1$.

This symmetry was expected as a consequence of the Onsager relations. In fact, the only asymmetric contribution in this analysis of heat and mass transfer coupling comes from the thermal conductivity, which has been dropped in Eq. (35).

As the factor inside the brackets of both Eqs. (34) and (35) is always positive, the sign of the ratios $M_{T S}$ and $Q_{S T}$ is determined by the signs of the gradients of the temperature and saturation ratio. If $M_{T S}=-1=Q_{S T}$, the contributions of these two gradients cancel each other out and then there is no net heat and mass fluxes within the porous medium. If $M_{T S}>0$ (and therefore $Q_{T S}>0$ ), then the contributions of both gradients to the heat and mass fluxes have the same sign; whereas, if $M_{T S}<0\left(Q_{T S}<0\right)$, the contributions of these two gradients partially cancel each other out (see Fig. 3).

We also conclude from Eq. (34) that the relative contribution of the temperature gradient to the mass flux is enhanced at low $\theta_{P}$ (the flat part of the isotherm) and is lowered at high $\theta_{P}$ (the steep part of the isotherm). Conversely, the saturation ratio gradient is more effective as a driving force of the heat flux at high $\theta_{P}$ than at low $\theta_{P}$ [see Eq. (36)].

Therefore, Eqs. (31) and (32) offer the possibility of assessing the adequate strategies for controlling heat and mass fluxes within porous media. In any process of twophase flow in unsaturated porous media (drying, for example) it enables the selection on the thermodynamic chart 
(made of different isotherms) of "the less energy-consuming path" or of "the faster path" or even the optima combination of both paths.

\subsection{Mass Flux Carried by the Vapor, Adsorbed, and Liquid Phases}

The mass flux through any cross section within an unsaturated porous medium is carried by the vapor and the adsorbed and liquid phases. From Eq. (19) we can assess the relative contribution of these phase flows as follows:

$$
\mathbf{J}_{\mathrm{mv}} / \mathbf{J}_{\mathrm{m}}=\left(\theta_{P} R_{\alpha} T\right)^{-1} \quad \text { with } \quad \theta_{P} R_{\alpha} T \geq 1
$$

We remark that Eq. (37) defines the weight of vapor mass flux in relation to the total mass flux. When the pores are completely filled with the liquid phase, the vapor flow is no longer possible. On the other hand, the isotherm slope is zero, therefore, leading to indetermination of the total mass flux as defined by Eq. (37). In this way, Eq. (37) cannot be applied to states corresponding to the isotherm plateau.

In the isotherms of Fig. 1, the low partial pressure range corresponds to mass adsorption on the internal surface of the pores (see Gregg and Sing, 1982). For this part of the isotherms $\theta_{P} R_{\alpha} T>1$ and from Eq. (30) we conclude that the mass flux corresponding to the vapor phase is very low and almost all the mass flux inside the porous medium corresponds to the adsorbed phase.

For the next part of the isotherm $\theta_{P} R_{\alpha} T \approx 1$, which means that almost all the mass flux is carried by the vapor diffusing inside the pores and driven by the partial pressure gradients. In fact, within this range, the filling of the narrowest pores begins by the liquid phase.

In the steeper part of the isotherm we have $\theta_{P} R_{\alpha} T \gg 1$. The contribution of the vapor phase to the total mass flux is very low as compared to that of the liquid phase. This part of the isotherm corresponds to the progressive filling of the pores with the liquid phase. The mass flux occurs mainly through the liquid, but vapor is also involved in the mass flux as described in Fig. 2, i.e., through evaporation at a meniscus, followed by vapor diffusion in the gas phase, condensation on the next meniscus, and then a transport through the liquid phase.

In this way, the sorption isotherms together with Eq. (37) provide important insight into the process of mass flow in unsaturated porous media.

\section{ISOTHERMAL NONSTATIONARY DIFFUSIVE MASS FLUX IN A POROUS MEDIUM}

In the following we analyze the influence of the isotherm slope $\theta_{P}$ on a nonstationary mass flux in an unsaturated porous medium.

By performing the time derivative of both members of the equation of the isotherm $\psi_{\alpha}=\psi_{\alpha}\left(P, T_{0}\right)$, with $P=$ $P_{s} S_{r}$ and considering mass conservation, Eq. (17) we obtain

$$
\theta_{P} P_{S}\left(\frac{\partial S_{r}}{\partial t}\right)_{T_{0}}=-\nabla \cdot \mathbf{J}_{\mathrm{m}}
$$

Equations (31) and (32) yield, under isothermal condition,

$$
\begin{aligned}
& \mathbf{J}_{\mathrm{m}}=-\frac{K_{\text {eff }}}{v} P_{s} \nabla S_{r} \\
& \mathbf{J}_{\mathbf{Q}}=\frac{K_{\text {eff }}(\Delta h)}{v \theta_{P} R_{\alpha} T} P_{s} \nabla S_{r}=-\frac{\Delta h}{\theta_{P} R_{\alpha} T} \mathbf{J}_{\mathbf{m}}
\end{aligned}
$$

Now, by combining Eqs. (38) and (39) we have

$$
\frac{\theta_{P}}{K_{\text {eff }}}\left(\frac{\partial S_{r}}{\partial t}\right)_{T_{0}}-\nabla^{2} S_{r}=0
$$

A solution to this equation, subjected to the initial condition $S_{r}(t=0, x, y, z)=\left(S_{r}\right)_{00}$ and to the boundary condition $S_{r}(t, x= \pm X, y= \pm Y, z= \pm Z)=\left(S_{r}\right)_{0}$, describes an isothermal mass flux inside a porous medium in an ambient at a fixed saturation ratio (or humidity) $\left(S_{r}\right)_{0}$, and has the form

$$
\begin{aligned}
& \Delta S_{r}=\left(\Delta S_{r}\right)_{0} \\
& \times \exp \left[-\frac{K_{\text {eff }} q^{2} t}{\theta_{P} V}+i \pi\left(\frac{x}{2 X}+\frac{y}{2 Y}+\frac{z}{2 Z}\right)\right]
\end{aligned}
$$

with

$$
\begin{aligned}
& q^{2}=\pi^{2}\left[\left(\frac{1}{2 X}\right)^{2}+\left(\frac{1}{2 Y}\right)^{2}+\left(\frac{1}{2 Z}\right)^{2}\right] \\
& \Delta S_{r}=S_{r}(t, x, y, z)-\left(S_{r}\right)_{00}
\end{aligned}
$$

and

$$
\left(\Delta S_{r}\right)_{0}=\left(S_{r}\right)_{0}-\left(S_{r}\right)_{00}
$$




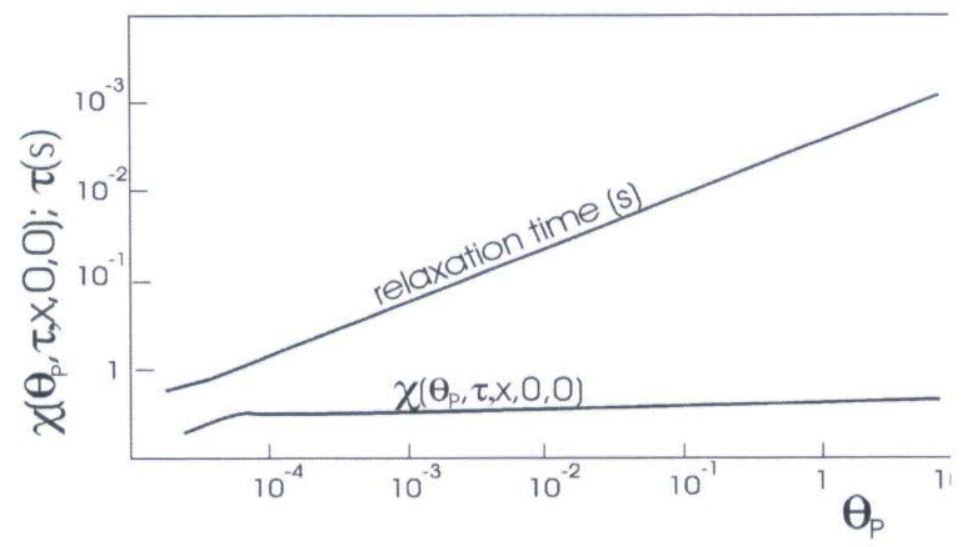

Figure 4. Relaxation time $\tau$ and $\chi\left(\theta_{P}, \tau, X, 0,0\right)$ as function of the isotherm slope $\theta_{P}$.

Taking into account (39) and (42) the $x$ component of the mass flux is

$$
\begin{aligned}
& \left(\mathbf{J}_{\mathbf{m}}\right)_{x}=-\frac{K_{\text {eff }}}{v} P_{s}\left(\Delta S_{r}\right)_{0} \frac{i \pi}{2 X} \\
& \times \exp \left[-\frac{K_{\text {eff }} q^{2} t}{v \theta_{P}}+i \pi\left(\frac{x}{2 X}+\frac{y}{2 Y}+\frac{z}{2 Z}\right)\right]
\end{aligned}
$$

The components $x$ and $z$ are obtained in an similar way from (39) and (42). In order to assess the influence of the isotherm slope $\theta_{P}$ on mass transfer we compare the mass fluxes as function of $\theta_{P}$ along the centerline $(x, 0,0)$ of the porous medium. From Eq. (43) we have

$$
\begin{aligned}
& \left(\mathbf{J}_{\mathbf{m}}\right)_{x, 0,0}=\frac{K_{\text {eff }}}{v} P_{s}\left(\Delta S_{r}\right)_{0} \\
& \times \frac{\pi}{2 X} \exp \left(-\frac{K_{\text {eff }} q^{2} t}{v \theta_{P}}\right) \sin \left(\frac{\pi x}{2 X}\right)
\end{aligned}
$$

Taking into account the value of the effective permeability, given by (A.3), this equation may be written as

$$
\left(\mathbf{J}_{\mathbf{m}}\right)_{x, 0,0}=\frac{K}{V} P_{s} \frac{\left(\Delta S_{r}\right)_{0}}{2 X} \chi\left(\theta_{P}, t, x, 0,0\right)
$$

where

$$
\chi\left(\theta_{P}, t, x, 0,0\right)=\tau^{-1} \frac{v \theta_{P}}{K q^{2}} \exp \left[-\tau^{-1} t\right] \sin \left(\frac{\pi x}{2 X}\right)
$$

the term

$$
\tau^{-1}=\left[1-\left(\theta_{P} R_{\alpha} T\right)^{-1}+K^{-1} D_{v} v \theta_{P}^{-1} R_{\alpha}^{-2} T^{-2}\right] \frac{K q^{2}}{v \theta_{P}}
$$

standing for the inverse of a "relaxation time" of the porous medium. The function $\chi(P, t, x, 0,0)$ describes the spatial and temporal evolution of the mass flux. As an example, for water at $280 \mathrm{~K}$ and taking $K=10^{-8} \cdot \mathrm{m}^{2}$ and $q=1 \mathrm{~m}^{-1}$, Fig. 4 shows the relaxation time and $\chi(P, \tau ; X, 0,0)$ as functions of the isotherm slope $\theta_{P}$.

The relaxation time increases almost linearly with $\theta_{P}$. This means that to the part of the isotherm curve with high $\theta_{P}$ - pores progressively filled with the liquid phase corresponds a large relaxation time, as should be expected. On the other hand, except for $\theta_{P}<10^{-4} \mathrm{~kg} \cdot \mathrm{Pa}^{-1} \cdot \mathrm{m}^{-3}$, the function $\chi(P, \tau ; X, 0,0)$ is practically constant and close to unity. This means that at a time equal to the relaxation time, the porous media exhibit the same spatial pattern of mass flux independently of the pore filling level.

In what concerns the isothermal heat flux, we conclude from the Eqs. (31) and (32) (taking $\nabla T=0$ ) that to a high isotherm slope corresponds a low ratio between heat and mass fluxes. In fact, Eq. (37) shows that the part of the vapor phase in the mass flux that carries the latent heat becomes progressively smaller with higher $\theta_{P}$. As the isothermal heat flow is totally coupled with the mass flow through these equations, its spatial and temporal behavior is analogous to that of the mass flux discussed above.

\section{CONCLUSIONS}

The sorption isotherms for fluids in porous media are an important source of information for the analysis of the heat and mass flux regimes within this media. The coupled heat and mass fluxes in unsaturated porous media can be related to the isotherm slope and other thermodynamic variables 
together with the fluid properties and the geometric properties of the porous medium.

Heat and mass fluxes in porous media are driven by the proper combination of partial pressure and emperature gradients. In this combination the isotherm slope is the variable that links the fluxes to the degree of saturation of the porous medium.

At subsaturation, i.e., low saturation ratio (or humidity), the partial pressure gradient drives the mass flux, which is also highly influenced by the temperature gradient. The mass flux is essentially composed of the vapor diffusing within the pores. At intermediate degrees of saturation ratio, heat and mass fluxes are strongly coupled, with the gradients of temperature and partial pressure giving contributions of the same order. The mass flux corresponds to two-phase flow characterized by successive steps consisting of evaporation, vapor diffusion through the gas phase, and condensation. Close to saturation (high $\theta_{P}$ ) the heat and mass fluxes remain strongly coupled, but in this range the temperature gradient becomes more important as a driving force for the mass flux.

The equations relating the heat and the mass fluxes to the temperature and partial pressure gradients may be useful for designing strategies for controlling the heat and mass fluxes within porous media.

Nonstationary isothermal mass flux has a relaxation time whose variation is almost linear with the isotherm slope. This result conforms with the fact that high isotherm slopes are associated to the part of the isotherm corresponding to pore filling, therefore, leading to a slow variation of the desorption (or sorption) mass fluxes.

It is found that the "effective permeability" coefficient of the porous medium to the mixed two-phase mass transfer - as it is defined in this work - is very close to the usual permeability coefficient for values of the isotherm slope higher than $10^{-3} \mathrm{~kg} \cdot \mathrm{Pa}^{-1} \cdot \mathrm{m}^{-3}$.

Although being a model based on experimental evidence, it should be the object of further experimental testing to validate the results presented here. The field of application is wide and embraces essentially the heat and mass transfer processes in unsaturated porous media (drying, impregnation, adsorption refrigeration, gas storage, civil engineering, chemical engineering, soil tilling, etc.).

\section{ACKNOWLEDGMENT}

The authors acknowledge the Geophysics Center of Evora for the support to this work.

\section{REFERENCES}

Ben Nasrallah, S. and Perre, P., Detailed study of a model of heat and mass transfer during convective drying of porous media, Int. J. Heat Mass Transfer, vol. 31, pp. 957-967, 1988.

De Boer, R., Thermodynamics of phase transitions in porous media, Appl. Mech. Rev., vol. 48, no. 10, pp. 613-622, 1995.

De Boer, R. and Kowalski, S. J., Thermodynamics of fluid-saturated porous media with a phase change, Acta Mechanica, vol. 109, pp. 167-189, 1995.

Gray, W. G., Macroscale equilibrium conditions for two-phase flow in porous media, Int. J. Multiphase Flow, vol. 26, pp. 467-501, 2000.

Gregg, S. J. and Sing, K. S. W., Adsorption, Surface Area and Porosity, 2nd end., Academic Press, New York, 1982.

Kondepudi, D. and Prigogine, I., Modern Thermodynamics From Heat Engines to Dissipative Structures, Wiley, New York, 1998.

Luikov, R. W., Systems of differential equations of heat and mass transfer in capillary-porous bodies (review), Int. J. Heat Mass Transfer, vol. 18, pp. 1-14, 1975.

Petit, F., Fichot, F., and Quintard, M., Écoulement diphasique en millieu poreux: Modele à non-équilibre local, Int. J. Therm. Sci., vol. 38, pp. 239-249, 1999.

Wang, C. Y. and Cheng, P., Multiphase flow and heat transfer in porous media (review), Adv. Heat Transfer, vol. 30, pp. 93-196, 1997.

Whitaker, S., Simultaneous heat, mass and momentum transfer in porous media: A theory of drying, Adv. Heat Transfer, vol. 13, pp. 119-203, 1977.

\section{APPENDIX: EFFECTIVE PERMEABILITY}

The definition of an effective permeability was made under the assumption that the liquid and vapor phases carry the mass flux. We neglect the part carried by the adsorbed phase, which may be important only at very low subsaturation (see Section 2.2). Then, from Eq. (37) we have for the vapor phase

$$
\mathbf{J}_{\mathrm{mv}} / \mathbf{J}_{\mathrm{m}}=\left(\theta_{P} R_{\mathrm{\alpha}} T\right)^{-1}
$$

and for the liquid phase 


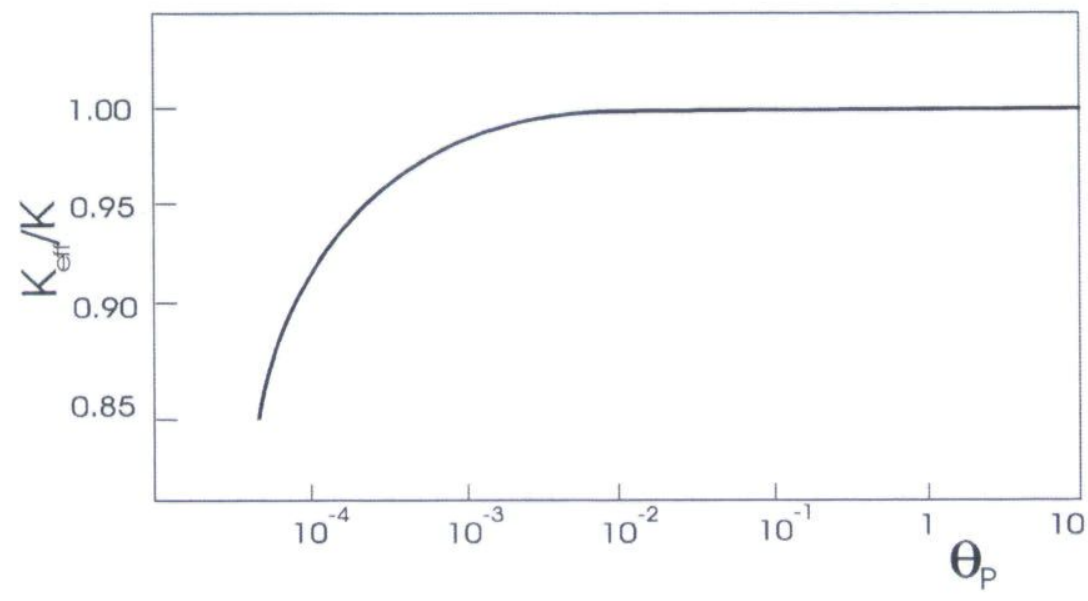

Figure 5. Effective permeability as function of the isotherm slope $\theta_{P}$.

$$
\mathbf{J}_{\mathrm{ml}} / \mathbf{J}_{\mathrm{m}}=1-\left(\theta_{P} R_{\alpha} T\right)^{-1}
$$

Therefore, by using Eqs. (9) and (10) for the vapor and liquid fluxes, and weighting the respective contributions, we get

$$
K_{\text {eff }} / K=1-\left(\theta_{P} R_{\alpha} T\right)^{-1}+K^{-1} D_{v} v \theta_{P}^{-1} R_{\alpha}^{-2} T^{-2}
$$

In Fig. 5, the ratio $K_{\text {eff }} / K$ is represented as a function of the isotherm slope. As happened with the function $\chi(\tau)$, the ratio $K_{\text {eff }} / K$ is practically equal to 1 for values of the isotherm slope $\theta_{P}>10^{-3} \mathrm{~kg} \cdot \mathrm{Pa}^{-1} \cdot \mathrm{m}^{-3}$. We note, again, the limitations of applicability of Eq. (37) referred to in Section 2. Therefore, Eq. (A.3) is not applicable to liquid-saturated porous media (corresponding to the isotherm plateau). 
Copyright of Journal of Porous Media is the property of Begell House Incorporated and its content may not be copied or emailed to multiple sites or posted to a listserv without the copyright holder's express written permission. However, users may print, download, or email articles for individual use. 